\title{
Characterizations of $N_{n c} e$-open and $N_{n c} e$-closed Functions
}

\author{
V. Sudha ${ }^{1_{*}}$, A. Vadivel ${ }^{2 \dagger}$ and S. Tamilselvan ${ }^{3 *}$ \\ Corresponding author: A. Vadivel \\ January 10, 2021
}

Article History: Received: 11 January 2021; Accepted: 27 February 2021; Published online: 5 April 2021

\begin{abstract}
The purpose of this paper is to introduce and investigate several new classes of functions called, $N_{n c} e$-open and $N_{n c} e$-closed functions in $N_{n c}$ topological spaces by using the concept of $N_{n c} e$-open sets. Several new characterizations and fundamental properties concerning of these new types of functions are obtained. Furthermore, these kinds of functions have strong application in the area of image processing and have very important applications in quantum particle physics, high energy physics and superstring theory.
\end{abstract}

Keywords and phrases: $N_{n c} e$-open sets, $N_{n c} e$-open functions and $N_{n c} e$-closed functions. AMS (2000) subject classification: 54A05, 54A10, 54C08

\section{Introduction}

Smarandache's neutrosophic system have wide range of real time applications for the fields of Computer Science, Information Systems, Applied Matheamatics, Artifical Intelligence, Mechanics, decision making, Medicine, Electrical \& Electronic, and Management Science etc [1, 2, 3, 4, 31, 32]. Topology is a classical subject, as a generalization topological spaces many types of topological spaces introduced over the year. Smarandache [25] defined the Neutrosophic set on three component Neutrosophic sets (T-Truth, F-Falsehood, I-Indeterminacy). Neutrosophic topological spaces (nts's) introduced by Salama and Alblowi [22]. Lellies Thivagar et.al. [12] was given the geometric existence of $N$ topology, which is a non-empty set equipped with $N$ arbitrary topologies. Lellis Thivagar et al. [13] introduced the notion of $N_{n}$-open (closed) sets and $N_{n}$ topological spaces. Al-Hamido [5] explore the possibility of expanding the concept of neutrosophic crisp topological spaces into $\mathrm{N}$-neutrosophic crisp topological spaces and investigate some of their basic properties. Several generalized forms of open and closed functions in topological spaces have been introduced and investigated over the course of years. Certainly, it is hard to say whether one form is more or less important than another. Functions and of course open and closed functions stand among the most important and most researched points in the whole of mathematical science. Various interesting problems arise when one considers openness and closeness. Its importance is significant in various areas of mathematics and related sciences. In 2008, Erdal Ekici [6] introduced a new class of generalized open sets called e-open sets and studied several fundamental and interesting properties of $e$-open sets and introduced a new class of continuous functions called $e$-continuous functions into the field of topology. In 2020, Vadivel and co-authors [27, 28] the concept of $N$ neutrosophic $\delta$-open, $N$-neutrosophic $\delta$-semiopen, $N$-neutrosophic $\delta$-preopen and $N$-neutrosophic $e$-open sets are introduced. In this paper, we will continue the study of related functions by involving $N_{n c} e$-open sets. The aim of this paper is to introduce and investigate several new types of $N_{n c}$-open and $N_{n c}$-closed functions in topological spaces via $N_{n c} e$-open sets. Some characterizations and several interesting properties of these functions are discussed. Additionally, these kinds of functions have strong application in the area of Image Processing and have very important applications in quantum particle physics, high energy physics and superstring theory.

\section{Preliminaries}

Salama and Smarandache [24] presented the idea of a neutrosophic crisp set in a set $X$ and defined the inclusion between two neutrosophic crisp sets, the intersection (union) of two neutrosophic crisp sets, the complement of a neutrosophic crisp set, neutrosophic crisp empty (resp., whole) set as more then two types. And they studied some properties related to nutrosophic crisp set operations. However, by selecting only one type, we define the inclusion, the intersection (union), and neutrosophic crisp empty (resp., whole) set again and discover a few properties.

*sudhasowjimath@gmail.com

†avmaths@gmail.com

\$tamil_au@yahoo.com ${ }^{1}$ Department of Mathematics, Periyar Arts College, Cuddalore, Tamil Nadu-607 001. ${ }^{2}$ Department of Mathematics, Government Arts College (Autonomous), Karur, Tamil Nadu-639 005; Department of Mathematics, Annamalai University, Annamalainagar, Tamil Nadu-608 002, and ${ }^{3}$ Mathematics Section (FEAT), Annamalai University, Annamalainagar, Tamil Nadu-608 002. 
Definition 2.1 Let $X$ be a non-empty set. Then $H$ is called a neutrosophic crisp set (in short, $n c s$ ) in $X$ if $H$ has the form

$H=\left(H_{1}, H_{2}, H_{3}\right)$, where $H_{1}, H_{2}$, and $H_{3}$ are subsets of $X$,

The neutrosophic crisp empty (resp., whole) set, denoted by $\phi_{n}\left(\right.$ resp., $\left.X_{n}\right)$ is an $n c s$ in $X$ defined by $\phi_{n}=(\phi, \phi, X)$

$\left.X_{n}=(X, X, \phi)\right)$. We will denote the set of all $n c s$ 's in $X$ as $n c S(X)$.

(resp.

In particular, Salama and Smarandache [23] classified a neutrosophic crisp set as the followings.

A neutrosophic crisp set $H=\left(H_{1}, H_{2}, H_{3}\right)$ in $X$ is called a neutrosophic crisp set of Type 1 (resp. $2 \& 3$ ) (in short, ncs-

Type

1 (resp. $2 \&$ 3) ), if it satisfies $H_{1} \cap H_{2}=H_{2} \cap H_{3}=H_{3} \cap H_{1}=\phi$ (resp. $H_{1} \cap H_{2}=H_{2} \cap H_{3}=H_{3} \cap H_{1}=\phi$ and $H_{1} \cup H_{2}$ $\cup H_{3}=X \& H_{1} \cap H_{2} \cap H_{3}=\phi$ and $\left.H_{1} \cup H_{2} \cup H_{3}=X\right)$. $n c S_{1}(X)\left(n c S_{2}(X)\right.$ and $\left.n c S_{3}(X)\right)$ means set of all ncs Type 1 (resp. 2 and 3 ).

Definition 2.2 Let $H=\left(H_{1}, H_{2}, H_{3}\right), M=\left(M_{1}, M_{2}, M_{3}\right) \in n c S(X)$. Then $H$ is said to be contained in (resp. equal to) $M$, denoted by $H \subseteq M$ (resp. $H=M)$, if $H_{1} \subseteq M_{1}, H_{2} \subseteq M_{2}$ and $H_{3} \supseteq M_{3}$ (resp. $H \subseteq M$ and $\left.M \subseteq H\right) ; H^{c}=\left(H_{3}, H_{2}^{c}, H_{1}\right)$; $H \cap M=(H 1 \cap M 1, H 2 \cap M 2, H 3 \cup M 3) ; H \cup M=(H 1 \cup M 1, H 2 \cup M 2, H 3 \cap M 3)$. Let $(A j) j \in J \subseteq n c S(X)$, where $H_{j}=$ $\left(H_{j} 1, H_{j} 2, H_{j} 3\right)$. Then $\cap H_{j}\left(\right.$ simply $\left.\cap H_{j}\right)=\left(\cap H_{j} 1, \cap H_{j} 2, \cup H_{j} 3\right) ; \cup H_{j}\left(\operatorname{simply} \cup H_{j}\right),=\left(\cup H_{j} 1, \cup H_{j} 2, \cap H_{j} 3\right)$.

$$
j \in J
$$

$j \in J$

The following are the quick consequence of Definition 2.2.

Proposition 2.1 [7] Let $L, M, O \in n c S(X)$. Then

(i) $\phi_{n} \subseteq L \subseteq X_{n}$,

(ii) if $L \subseteq M$ and $M \subseteq O$, then $L \subseteq O$,

(iii) $L \cap M \subseteq L$ and $L \cap M \subseteq M$,

(iv) $L \subseteq L \cup M$ and $M \subseteq L \cup M$,

(v) $L \subseteq M$ iff $L \cap M=L$,

(vi) $L \subseteq M$ iff $L \cup M=M$.

Likewise the following are the quick consequence of Definition 2.2.

Proposition 2.2 [7] Let $L, M, O \in n c S(X)$. Then

(i) $L \cup L=L, L \cap L=L$ (Idempotent laws),

(ii) $L \cup M=M \cup L, L \cap M=M \cap L$ (Commutative laws),

(iii) (Associative laws) : $L \cup(M \cup O)=(L \cup M) \cup O, L \cap(M \cap O)=(L \cap M) \cap O$,

(iv) (Distributive laws:) $L \cup(M \cap O)=(L \cup M) \cap(L \cup O), L \cap(M \cup O)=(L \cap M) \cup(L \cap O)$,

(v) (Absorption laws) : $L \cup(L \cap M)=L, L \cap(L \cup M)=L$,

(vi) (DeMorgan's laws) : $(L \cup M)^{c}=L^{c} \cap M^{c},(L \cap M)^{c}=L^{c} \cup M^{c}$,

(vii) $\left(L^{c}\right)^{c}=L$,

(viii) (a) $L \cup \phi_{n}=L, L \cap \phi_{n}=\phi_{n}$,

(b) $L \cup X_{n}=X_{n}, L \cap X_{n}=L$,

(c) $X_{n}^{c}=\phi, \phi^{c}{ }_{n}=X_{n}$

(d) in general, $L \cup L^{c}=\square \quad X_{n}, L \cap L^{c}=\square \quad \phi_{n}$.

Proposition 2.3 [7] Let $L \in n c S(X)$ and let $\left(L_{j}\right)_{j} \in J \subseteq n c S(X)$. Then

(i) $\left(\cap L_{j}\right)^{c}=\bigcup L_{j}^{c},\left(\bigcup L_{j}\right)^{c}=\bigcap_{L c j}$,

(ii) $L \cap\left(\cup L_{j}\right)=\cup\left(L \cap L_{j}\right), L \cup\left(\cap L_{j}\right)=\cap\left(L \cup L_{j}\right)$.

Definition 2.3 [23] A neutrosophic crisp topology (briefly, ncts) on a non-empty set $X$ is a family $\tau$ of $n c$ subsets of $X$ satisfying the following axioms

(i) $\phi_{n}, X_{n} \in \tau$.

(ii) $H_{1} \cap H_{2} \in \tau \forall H_{1} \& H_{2} \in \tau$.

(iii) $\cup H_{a} \in \tau$, for any $\left\{H_{a}: a \in J\right\} \subseteq \tau$.

$a$

Then $(X, \tau)$ is a neutrosophic crisp topological space (briefly, ncts ) in $X$. The $\tau$ elements are called neutrosophic crisp open sets (briefly, ncos) in $X$. A ncs $C$ is closed set (briefly, nccs) iff its complement $C^{c}$ is $n c o s$.

Definition 2.4 [5] Let $X$ be a non-empty set. Then ${ }_{n c} \tau_{1},{ }_{n c} \tau_{2}, \cdots,{ }_{n c} \tau_{N}$ are $N$-arbitrary crisp topologies defined on $X$ and the collection $N_{n c} \tau=\left\{A \subseteq X: A=\left(\cup H_{j}\right) \cup\left(\cap L_{j}\right), H_{j}, L_{j} \in{ }_{n c} \tau_{j}\right\}$ is called $N$ neutrosophic crisp (briefly, $N_{n c}$ )-topology on

$X$ if the axioms are satisfied:

$\begin{array}{cc}N & N \\ j=1 & j=1\end{array}$


(i) $\phi_{n}, X_{n} \in N_{n c} \tau$.

(ii) $\bigcup_{j=1}^{\infty} A_{j} \in \quad \tau \forall\left\{A_{j}\right\}_{j=1}^{\infty} \in N n c \quad N n c \tau$.

$j=1$

$n j=1$

(iii) $\cap A j \in N n c \tau \forall\{A j\} n j=1 \in N n c \tau$.

Then $\left(X, N_{n c} \tau\right)$ is called a $N_{n c}$-topological space (briefly, $\left.N_{n c} t s\right)$ on $X$. The $N_{n c} \tau$ elements are called $N_{n c}$-open sets $\left(N_{n c} O s\right)$ on $X$ and its complement is called $N_{n c}$-closed sets $\left(N_{n c} c s\right)$ on $X$. The elements of $X$ are known as $N_{n c}$-sets $\left(N_{n c} s\right)$ on $X$. Definition 2.5 [5] Let $\left(X, N_{n c} \tau\right)$ be any $N_{n c} t s$. Let $H$ be an $N_{n c} s$ in $\left(X, N_{n c} \tau\right)$. Then $H$ is said to be a $N_{n c}$-regular open [26] set (briefly, $N_{n c} r o s$ ) if $H=N_{n c} i n t\left(N_{n c} c l(H)\right)$. The complement of an $N_{n c} r o s$ is called an $N_{n c}$-regular closed set (briefly, $N_{n c} r c s$ ) in $X$.

The family of all $N_{n c} r o s$ (resp. $N_{n c} r c s$ ) of $X$ is denoted by $N_{n c} R O S(X)$ (resp. $N_{n c} R C S(X)$ ).

Definition 2.6 [27] A set $H$ is said to be a

(i) $N_{n c} \delta$ interior of $H$ (briefly, $N_{n c} \operatorname{\delta int}(H)$ ) is defined by $N_{n c} \operatorname{sint}(H)=\cup\left\{A: A \subseteq H \& A\right.$ is a $\left.N_{n c} r o s\right\}$.

(ii) $N_{n c} \delta$ closure of $H$ (briefly, $N_{n c} \delta c l(H)$ ) is defined by $N_{n c} \delta c l(H)=\bigcup\left\{x \in X: N_{n c} \operatorname{int}\left(N_{n c} c l(L)\right) \cap H=\square \phi, x \in L \&\right.$ $L$ is a $\left.N_{n c} O S\right\}$.

Definition 2.7 A set $H$ is said to be a

(i) $N_{n c} \delta$ - open (briefly, $N_{n c} \delta o$ ) set [27] if $H=N_{n c} \operatorname{\delta int}(H)$.

(ii) $N_{n c} e$-open (briefly, $N_{n c} e o$ ) set [28] if $H \subseteq N_{n c} c l\left(N_{n c} \delta \operatorname{int}(H)\right) \cup N_{n c} i n t\left(N_{n c} \delta c l(H)\right)$.

The complement of an $N_{n c} \delta o s$ (resp. $N_{n c} e o s$ ) is called an $N_{n c} \delta$ (resp. $N_{n c} e$ ) closed set (briefly, $N_{n c} \delta c s$ (resp. $N_{n c} e c s$ )) in $X$.

The family of all $N_{n c} e o s$ (resp. $\left.N_{n c} e c s\right)$ of $X$ containing a point $x \in X$ is denoted by $N_{n c} e O S(X, x)\left(\operatorname{resp} . N_{n c} e C S(X, x)\right)$. The family of all $N_{n c} \delta o s$ (resp. $N_{n c} \delta c s, N_{n c} e o s$ and $N_{n c} e c s$ ) of $X$ is denoted by $N_{n c} \delta O S(X)\left(\operatorname{resp} . N_{n c} \delta C S(X), N_{n c} e O S(X)\right.$ and $\left.N_{n c} e C S(X)\right)$.

Definition 2.8 A function $f:\left(X, N_{n c} \tau\right) \rightarrow\left(Y, N_{n c} \tau^{*}\right)$ is said to be $N_{n c}$-continuous (briefly, $\left.N_{n c} e C t s\right)$ [29], if $f^{-1}(V)$ is $N_{n c} e o$ in $X$ for every $N_{n c} O$ set $V$ of $Y$.

Definition 2.9 A space $\left(X, N_{n c} \tau\right)$ is said to be:

(i) $N_{n c} e-T_{1}$ [30] if for each pair of distinct points $x$ and $y$ of $X$, there exist $N_{n c} e o$ sets $A$ and $B$ containing $x$ and $y$, respectively, such that $x / \in B$ and $y / \in A$.

(ii) $N_{n c} e-T_{2}$ [30] if for each pair of distinct points $x$ and $y$ in $X$, there exist disjoint $N_{n c} e o$ sets $A$ and $B$ in $X$ such that $x \in A$ and $y \in B$.

Definition 2.10 A space $\left(X, N_{n c} \tau\right)$ is said to be:

(i) $N_{n c} e$-compact [30] if every cover of $X$ by $N_{n c} e o$ sets has a $N_{n c}$ finite sub cover.

(ii) $N_{n c} e$-Lindelo"f [30] if every cover of $X$ by $N_{n c} e o$ sets has a countable subcover.

Definition 2.11 A space $\left(X, N_{n c} \tau\right)$ is said to be $N_{n c} e$-connected [30] if $X$ cannot be written as the union of two nonempty disjoint $N_{n c} e o$ sets.

3 Characterizations of $N_{n c} e$-open and $N_{n c} e$-closed functions

In this section, we obtain some characterizations and several properties concerning $N_{n c} e$-open functions and $N_{n c} e$ closed functions via $N_{n c} e O$ and $N_{n c} e c$ sets.

Definition 3.1 A function $f:\left(X, N_{n c} \tau\right) \rightarrow\left(Y, N_{n c} \tau^{*}\right)$ is said to be $N_{n c} e$-open (briefly. $\left.N_{n c} e O\right)$ if $f(U) \in N_{n c} e O S(Y)$ for every $N_{n c} O$ set $U$ in $X$.

Theorem 3.1 A function $f:\left(X, N_{n c} \tau\right) \rightarrow\left(Y, N_{n c} \tau^{*}\right)$ is $N_{n c} e O$ iff for each $x \in X$ and each $N_{n c} O$ set $U$ in $X$ with $x \in U$, there exists a set $V \in N_{n c} e O S(Y)$ containing $f(x)$ such that $V \subseteq f(U)$.

Proof. The proof is follows immediately from Definition 3.1.

Theorem 3.2 Let $f:\left(X, N_{n c} \tau\right) \rightarrow\left(Y, N_{n c} \tau^{*}\right)$ be $N_{n c} e O$. If $V \subseteq Y$ and $M$ is a $N_{n c} c$ set of $X$ containing $f^{-1}(V)$, then there exists a set $F \in N_{n c} e C S(Y)$ containing $V$ such that $f^{-1}(F) \subseteq M$.

Proof. Let $F=Y-f(X-M)$. Then, $F \in N_{n c} e C S(Y)$, since $f^{-1}(V) \subseteq M$, we have, $f(X-M) \subseteq(Y-V)$ and so $V \subseteq F$. Also $f^{-1}(F)=X-f^{-1}(f(X-M)) \subseteq X-(X-M)=M$.

Theorem 3.3 A function $f:\left(X, N_{n c} \tau\right) \rightarrow\left(Y, N_{n c} \tau^{*}\right)$ is $N_{n c} e O \operatorname{iff} f\left(N_{n c} \operatorname{int}(A)\right) \subseteq N_{n c} e i n t(f(A))$, for every $A \subseteq X$.

Proof. Let $A \subseteq X$ and $x \in N_{n c} i n t(A)$. Then there exists an $N_{n c} O$ set $U_{x}$ in $X$ such that $x \in U_{x} \subseteq A$. Now $f(x) \in f\left(U_{x}\right) \subseteq$ $f(A)$, since $f$ is $N_{n c} e O, f\left(U_{x}\right) \in N_{n c} e O S(Y)$. Then, $f(x) \in N_{n c} e i n t(f(A))$. Thus $f\left(N_{n c} \operatorname{int}(A)\right) \subseteq N_{n c} e \operatorname{int}(f(A))$. Conversely, let $U$ be an $N_{n c} O$ set in $X$. Then by assumption, $f\left(N_{n c} \operatorname{int}(U)\right) \subseteq N_{n c} \operatorname{eint}(f(U))$. Since $N_{n c} \operatorname{eint}(f(U)) \subseteq f(U), f(U)=$ $N_{n c} \operatorname{eint}(f(U))$. Thus $f(U) \in N_{n c} e O S(Y)$. So $f$ is $N_{n c} e O$.

Remark 3.1 The equality in the Theorem 3.3 need not be true as shown in the following Example.

Example 3.1 Let $X=\{a, b, c, d, e\},{ }_{n c} \tau_{1}=\left\{\phi_{N}, X_{N}, A, B, C\right\},{ }_{n c} \tau_{2}=\left\{\phi_{N}, X_{N}\right\} . A=\langle\{c\},\{\phi\},\{a, b, d, e\}\rangle, B=\langle\{a, b\},\{\phi\},\{c, d, e\}$ $C=\langle\{a, b, c\},\{\phi\},\{d, e\}\rangle$, then we have $2_{n c} \tau=\left\{\phi_{N}, X_{N}, A, B, C\right\}$. Define $f:\left(X, 2_{n c} \tau\right) \rightarrow\left(X, 2_{n c} \tau\right)$ be an identity function. 
Then $f$ is a $2_{n c} e O$. Let $U=\langle\{c, d\},\{\phi\},\{a, b, e\}\rangle \subseteq X$. Then $f\left(2_{n c} \operatorname{int}(U)\right)=f\left(2_{n c} \operatorname{int}(\langle\{c, d\},\{\phi\},\{a, b, e\}\rangle)\right)=$ $f(\langle\{c\},\{\phi\},\{a, b, d, e\}\rangle)=\langle\{c\},\{\phi\},\{a, b, d, e\}\rangle$. But $2_{n c} \operatorname{eint}(f(U))=2_{n c} \operatorname{eint}(f(\langle\{c, d\},\{\phi\},\{a, b, e\}\rangle))=$ $2_{n c} \operatorname{eint}\left(\left\langle\{c, d\},\{\phi\},\left\{a, b, e\langle\{c, d\},\{\phi\},\{a, b, e\}\rangle\right.\right.\right.$. Thus $f\left(2_{n c} \operatorname{int}(U)\right)=2 \square n c e i n t(f(U))$.

Theorem 3.4 A function $f:\left(X, N_{n c} \tau\right) \rightarrow\left(Y, N_{n c} \tau^{*}\right)$ is $N_{n c} e O$ iff $N_{n c} \operatorname{int}\left(f^{-1}(B)\right) \subseteq f^{-1}\left(N_{n c} e i n t(B)\right)$ for every $B \subseteq Y$.

Proof. Let $B$ be any $N_{n c}$ set of $Y$. Then $f\left(N_{n c} \operatorname{int}\left(f^{-1}(B)\right)\right) \subseteq f\left(f^{-1}(B)\right) \subseteq B$. But $f\left(N_{n c} i n t\left(f^{-1}(B)\right)\right) \in N_{n c} e O S(Y)$, since $N_{n c} \operatorname{int}\left(f^{-1}(B)\right)$ is $N_{n c} O$ in $X$ and $f$ is $N_{n c} e O$. Hence, $f\left(N_{n c} \operatorname{int}\left(f^{-1}(B)\right)\right) \subseteq N_{n c} e i n t(B)$. Therefore $N_{n c} i n t\left(f^{-1}(B)\right) \subseteq$ $f^{-1}\left(N_{n c} \operatorname{eint}(B)\right)$.

Conversely, let $A$ be any $N_{n c}$ set of $X$. Then $f(A) \subseteq Y$. Hence by assumption, we have, $N_{n c} \operatorname{int}(A) \subseteq N_{n c} \operatorname{int}\left(f^{-1}(f(A))\right.$ $\left.\subseteq f^{-1}\left(N_{n c} e i n t(f(A))\right)\right)$. Thus, $f\left(N_{n c} \operatorname{int}(A)\right) \subseteq N_{n c} \operatorname{eint}(f(A))$, for every $A \subseteq X$. Hence, by Theorem 3.3, $f$ is $N_{n c} e O$. Theorem 3.5 A function $f:\left(X, N_{n c} \tau\right) \rightarrow\left(Y, N_{n c} \tau^{*}\right)$ is $N_{n c} e o$ iff $f^{-1}\left(N_{n c} e c l(B)\right) \subseteq N_{n c} c l\left(f^{-1}(B)\right)$ for every $B \subseteq Y$.

Proof. Suppose that $f$ is $N_{n c} e O$ and $B \subseteq Y$ and let $x \in f^{-1}\left(N_{n c} e c l(B)\right)$. Then, $f(x) \in N_{n c} e c l(B)$. Let $U$ be an $N_{n c} O$ set in $X$ such that $x \in U$. Since $f$ is $N_{n c} e o$, then $f(U) \in N_{n c} e O S(Y)$. Therefore $B \cap f(U) \square=\phi$. Then, $U \cap f^{-1}(B) \square=\phi$. Hence $x \in N_{n c} c l\left(f^{-1}(B)\right)$. Therefore we have $f^{-1}\left(N_{n c} e c l(B) \subseteq N_{n c} c l\left(f^{-1}(B)\right)\right.$.

Conversely, let $B \subseteq Y$, then $(Y-B) \subseteq Y$. By assumption, $f^{-1}\left(N_{n c} e c l(Y-B)\right) \subseteq N_{n c} c l\left(f^{-1}(Y-B)\right)$ this implies, $X-$ $N_{n c} c l\left(f^{-1}(Y-B)\right) \subseteq X-f^{-1}\left(N_{n c} e c l(Y-B)\right)$. Hence $X-N_{n c} c l\left(X-f^{-1}(B)\right) \subseteq f^{-1}\left(\left(Y-N_{n c} e c l(Y-B)\right)\right)$.

Now $X-N_{n c} c l\left(X-f^{-1}(B)\right)=N_{n c} \operatorname{int}\left(X-\left(X-f^{-1}(B)\right)=N_{n c} \operatorname{int}\left(f^{-1}(B)\right)\right)$. Then, we have $Y-N_{n c} e c l(Y-B)=N_{n c}$ eint $(Y-$ $(Y-B))=N_{n c} e i n t(B)$. Then $N_{n c} \operatorname{int}\left(f^{-1}(B)\right) \subseteq f^{-1}\left(N_{n c} e i n t(B)\right)$. By Theorem 3.4 we have $f$ is $N_{n c} e o$.

Now we introduce some characterizations concerning $N_{n c} e$-closed functions.

Definition 3.2 A function $f:\left(X, N_{n c} \tau\right) \rightarrow\left(Y, N_{n c} \tau^{*}\right)$ is said to be $N_{n c} e$-closed (briefly, $\left.N_{n c} e C\right)$ if $f(M) \in N_{n c} e C S(Y)$ for every $N_{n c} c$ set $M$ in $X$.

Example 3.2 Let $X=\{a, b, c, d, e\},{ }_{n c} \tau_{1}=\left\{\phi_{N}, X_{N}, A, B, C\right\},{ }_{n c} \tau_{2}=\left\{\phi_{N}, X_{N}\right\} . A=\langle\{c\},\{\phi\},\{a, b, d, e\}\rangle, B=\langle\{a, b\},\{\phi\},\{c, d, e\}$ $C=\langle\{a, b, c\},\{\phi\},\{d, e\}\rangle$, then we have $2_{n c} \tau=\left\{\phi_{N}, X_{N}, A, B, C\right\}$. Define $f:\left(X, 2_{n c} \tau\right) \rightarrow\left(X, 2_{n c} \tau\right)$ be an identity function.

Then $f$ is a $2_{n c} e C$.

Theorem 3.6 A function $f:\left(X, N_{n c} \tau\right) \rightarrow\left(Y, N_{n c} \tau^{*}\right)$ is $N_{n c} e C$ iff $N_{n c} e c l(f(A)) \subseteq f\left(N_{n c} c l(A)\right)$ for every $A \subseteq X$.

Proof. Let $f$ be $N_{n c} e C$ function and let $A$ be any $N_{n c}$ set of $X$. Then $f\left(N_{n c} c l(A)\right) \in N_{n c} e C S(Y)$. But $f(A) \subseteq f\left(N_{n c} c l(A)\right)$. Then $N_{n c} e c l(f(A)) \subseteq f\left(N_{n c} c l(A)\right)$.

Conversely, let $A$ be a $N_{n c} c$ set of $X$. Then by assumption, $N_{n c} e c l(f(A)) \subseteq f\left(N_{n c} c l(A)\right)=f(A)$. This shows that $f(A) \in$ $N_{n c} e C S(X)$. Hence $f$ is $N_{n c} e C$.

Corollary 3.1 Let $f:\left(X, N_{n c} \tau\right) \rightarrow\left(Y, N_{n c} \tau^{*}\right)$ be $N_{n c} e C$ and let $A \subseteq X$. Then, $N_{n c} \operatorname{eint}\left(N_{n c} e c l(f(A)) \subseteq f\left(N_{n c} c l(A)\right)\right)$.

Theorem 3.7 Let $f:\left(X, N_{n c} \tau\right) \rightarrow\left(Y, N_{n c} \tau^{*}\right)$ be a surjective function. Then $f$ is $N_{n c} e C$ iff for each subset $B$ of $Y$ and each $N_{n c} O$ set $U$ in $X$ containing $f^{-1}(B)$, there exists a set $V \in N_{n c} e O S(Y)$ containing $B$ such that $f^{-1}(V) \subseteq U$.

Proof. Let $V=Y-f(X-U)$, then $V \in N_{n c} e O S(Y)$. Since $f^{-1}(B) \subseteq U$, then we have $f(X-U) \subseteq Y-B$ so $B \subseteq V$. Also, $f^{-1}(V)=X-f^{-1}(f(X-U)) \subseteq X-(X-U)=U$.

Conversely, let $M$ be a $N_{n c} c$ set in $X$ and $y \in Y-f(M)$. Then, $f^{-1}(y) \subseteq X-f^{-1}(f(M)) \subseteq X-M$ and $X-M$ is $N_{n c} O$ in $X$. Hence by assumption, there exists a set $V_{y} \in N_{n c} e O S(Y, y)$ such that $f^{-1}\left(V_{y}\right) \subseteq X-M$. This implies that $y \in V_{y} \subseteq Y-$ $f(M)$. Thus $Y-f(M)=\cup\left\{V_{y}: y \in Y-f(M)\right\}$. Hence $Y-f(M) \in N_{n c} e O S(Y)$. Thus $f(M) \in N_{n c} e C S(Y)$.

Theorem 3.8 Let $f: X \rightarrow Y$ be a bijective. Then the following are equivalent:

(i) $f$ is $N_{n c} e C$, (ii) $f$ is $N_{n c} e O$,

(iii) $f^{-1}$ is $N_{n c} e C t s$.

Proof. (i) $\Rightarrow$ (ii): Let $U$ be an $N_{n c} o$ set of $X$. Then $X-U$ is $N_{n c} c$ in $X$. By (i), $f(X-U) \in N_{n c} e C S(Y)$. But $f(X-U)=f(X)$ $-f(U)=Y-f(U)$. Thus $f(U) \in N_{n c} e O S(Y)$.

(ii) $\Rightarrow$ (iii): Let $U$ be an $N_{n c} o$ set of $X$. Since $f$ is $N_{n c} e o$. Then, $f(U)=\left(f^{-1}\right)^{-1}(U) \in N_{n c} e O S(Y)$. Hence $f^{-1}$ is $N_{n c} e C t s$.

(iii) $\Rightarrow$ (i): Let $M$ be an arbitrary $N_{n c} c$ set in $X$. Then $X-M$ is $N_{n c} o$ in $X$. Since $f^{-1}$ is $N_{n c} e C t s$, then $\left(f^{-1}\right)^{-1}(X-M) \in$ $N_{n c} e O S(Y)$. But $\left(f^{-1}\right)^{-1}(X-M)=f(X-M)=Y-f(M)$, thus $f(M) \in N_{n c} e C S(Y)$.

Theorem 3.9 If $f:\left(X, N_{n c} \tau\right) \rightarrow\left(Y, N_{n c} \tau^{*}\right)$ is $N_{n c} e O$ bijection. Then the following hold:

(i) If $X$ is $N_{n c} T_{1}$ then $Y$ is $N_{n c} e-T_{1}$. (ii) If $X$ is $N_{n c} T_{2}$ then $Y$ is $N_{n c} e-T_{2}$.

Proof. (i) Let $y_{1}$ and $y_{2}$ be any distinct points in $Y$. Then there exist $x_{1}$ and $x_{2}$ in $X$ such that $f\left(x_{1}\right)=y_{1}$ and $f\left(x_{2}\right)=y_{2}$. Since $X$ is $N_{n c} T_{1}$ then, there exist two $N_{n c} o$ sets $U$ and $V$ in $X$ with $x_{1} \in U, x_{2} \in / U$ and $x_{2} \in V, x_{1} \in / V$. Now $f(U)$ and $f(V)$ are $N_{n c} e o$ in $Y$ with $y_{1} \in f(U), y_{2} \in / f(U)$ and $y_{2} \in f(V), y_{1} \in / f(V)$.

(ii) It is similar to (i). Thus is omitted.

Theorem 3.10 If $f:\left(X, N_{n c} \tau\right) \rightarrow\left(Y, N_{n c} \tau^{*}\right)$ is $N_{n c} e O$ bijective. Then the following properties are hold:

(i) If $Y$ is $N_{n c} e$-compact, then $X$ is compact.

(ii) If $Y$ is $N_{n c} e$-Lindel $o " \mathrm{f}$, then $X$ is Lindel $\%$ " f. 
Proof. (i) Let $U_{1}=\left\{U_{\lambda}: \lambda \in \Delta\right\}$ be an $N_{n c} o$ cover of $X$. Then $K_{1}=\left\{f\left(U_{\lambda}\right): \lambda \in \Delta\right\}$ is a cover of $Y$ by $N_{n c} e o$ sets in $Y$. Since $Y$ is $N_{n c} e$-compact, Then $K_{1}$ has a $N_{n c}$ finite subcover $K_{2}=\left\{f\left(U_{\lambda 1}\right), f\left(U_{\lambda 2}\right), \cdots, f\left(U_{\lambda n}\right)\right\}$ for $Y$. Then $U_{2}=\left\{U_{\lambda 1}, U_{\lambda 2}, \cdots\right.$ ,$\left.U_{\lambda n}\right\}$ is a $N_{n c}$ finite subcover of $U$ for $X$.

(ii) It is similar to (i). Thus is omitted.

Theorem 3.11 If a function $f:\left(X, N_{n c} \tau\right) \rightarrow\left(Y, N_{n c} \tau^{*}\right)$ is an $N_{n c} e O$ surjective and $Y$ is $N_{n c} e$-connected. Then $X$ is $N_{n c}$ connected.

Proof. Suppose that $X$ is not $N_{n c}$-connected. Then there exist two non-empty disjoint $N_{n c} O$ sets $U$ and $V$ in $X$ such that $X=U \cup V$. Then $f(U)$ and $f(V)$ are non-empty disjoint $N_{n c} e o$ sets in $Y$ with $Y=f(U) \cup f(V)$ which contradicts the fact that $Y$ is $N_{n c} e$ connected.

4 Conclusion

Generalized open and closed sets play a very a prominent role in general Topology and it applications. And many topologists worldwide are focusing their researches on these topics and this mounted to many important and useful results. Indeed a significant theme in General Topology, Real analysis and many other branches of mathematics concerns the variously modified forms of continuity, separation axioms etc., by utilizing generalized open and closed sets. One of the well-known notions and that expected it will has a wide applying in physics and Topology and their applications is the notion of $N_{n c} e$-open sets. The importance of general topological spaces rapidly increases in both the pure and applied directions; it plays a significant role in data mining [21]. One can observe the influence of general topological spaces also in computer science and digital topology $[8,9,10]$, computational topology for geometric and molecular design [14], particle physics, high energy physics, quantum physics, and Superstring theory [11, 15, 16, $17,18,19,20]$. In this paper we have introduced and investigated the notions of new classes of functions which may have very important applications in quantum particle physics, high energy physics and superstring theory. Furthermore, the fuzzy topological version of the concepts and results introduced in this paper are very important. Since El-Naschie has shown that the notion of fuzzy topology has very important applications in quantum particle physics especially in related to both string theory and $\epsilon^{\infty}$ theory.

References

1. M. Abdel-Basset, V. Chang, M. Mohamed and F. Smarandche, A Refined Approach for Forecasting Based on Neutrosophic Time Series, Symmentry, 11 (4) (2019) 457.

2. M. Abdel-Basset, G. Manogaran, A. Gamal and V. Chang, A Novel Intelligent Medical Decision Support Model Based on Soft Computing and IoT, IEEE Internet of Things Journal, (2019).

3. M. Abdel-Basset, and M. Mohamed, A novel and powerful framework based on neutrosophic sets to aid patients with cancer, Future Generation Computer Systems, 98 (2019) 144-153.

4. M. Abdel-Basset, A. Gamal, G. Manogaran and H. V. Long A novel group decision making model based on neutrosophic sets for heart disease diagnosis, Multimedia Tools and Applications, (2019) 1-26.

5. R. K. Al-Hamido, T. Gharibah, S. Jafari and F. Smarandache, On neutrosophic crisp topology via Ntopology, Neutrosophic Sets and Systems, 23 (2018), 96-109.

6. Erdal Ekici, On e-open sets, $\mathrm{DP}^{\star}$-sets and $\mathrm{DP} \epsilon{ }^{*}$-sets and decomposition of continuity, The Arabian Journal for Science and Engineering, 33 (2008) 271-282.

7. K. Hur, P. K. Lim, J. G. Lee and J. Lee, The category of neutrosophic crisp sets, Annals of Fuzzy mathematics and Informatics, 14 (1) (2017), 43-54.

8. E. D. Khalimsky, R. Kopperman and P. R. Meyer, Computer graphics and connected topologies on finite ordered sets, Topology and its Applications, 36 (1990), 117.

9. T. Y. Kong, R. Kopperman and P. R. Meyer, A topological approach to digital topology, Amer. Math. Monthly, 98 (10) (1991), 901917.

10. V. Kovalesky and R. Kopperman, Some topology-based imaged processing algorithms, Ann. NY. Acad. Sci, 728 (1) (1994), 174182.

11. G. Landi, An introduction to noncommutative spaces and their geometrics (lecture notes in physics), New York, SpringerVerlag, (1997).

12. M. Lellis Thivagar, V. Ramesh, M D. Arockia, On new structure of N-topology, Cogent Mathematics (Taylor and Francis), 3 (2016):1204104.

13. M. Lellis Thivagar, S. Jafari, V. Antonysamy and V.Sutha Devi, The ingenuity of neutrosophic topology via $N$-topology, Neutrosophic Sets and Systems, 19 (2018), 91-100.

14. E. L. F. Moore and T. J. Peters, Computational topology for geometric design and molecular design, In: D.R. Ferguson, T. J. Peters, editors, Mathematics in industry challenges and frontiers, 2003, SIAM, 2005. 
15. M. S. El-Naschie, O. E. Rossler and G. Oed, Information and diffusion in quantum physics. Chaos, Solitons \& Fractals, 7 (5)

16. (1996),

17. M. S. El-Naschie, On the uncertainty of Cantorian geometry and the two-slit experiment, Chaos, Solitons \& Fractals, 9 (1998), 51729.

18. M. S. El-Naschie, On the unification of heterotic strings, $M$ theory and $\epsilon^{\infty}$ theory, Chaos, Solitons \& Fractals, 11 (14) (2000), 23972408.

19. M. S. El-Naschie, Quantum gravity from descriptive set theory, Chaos, Solitons \& Fractals, 19 (5) (2004), 13391344.

20. M. S. El-Naschie, Quantum gravity, Clifford algebras, fuzzy set theory and the fundamental constants of nature, Chaos, Solitons \& Fractals, 20 (3) (2004), 437450.

21. M. S. El-Naschie, The two slit experiment as the foundation of E-infinity of high energy physics, Chaos, solitons \& Fractals, 25 (3) (2005), 509514.

22. Z. Pawlak, Rough sets: theoretical aspects of reasoning about data, System theory, knowledge engineering and problem solving, Dordrecht, Kluwer, 9 (1991).

23. A. A. Salama and S. A. Alblowi, Generalized neutrosophic set and generalized neutrosophic topological spaces, Journal computer sci. engineering, 2 (7) (2012), 31-35.

24. A. A. Salama, F. Smarandache and V. Kroumov, Neutrosophic crisp sets and neutrosophic crisp topological spaces, Neutrosophic Sets and Systems, 2 (2014), 25-30.

25. A. A. Salama and F. Smarandache, Neutrosophic crisp set theory, Educational Publisher, Columbus, Ohio, USA, 2015.

26. F. Smarandache, Neutrosophy and neutrosophic logic, First International Conference on Neutrosophy, Neutrosophic Logic, Set, Probability, and Statistics, University of New Mexico, Gallup, NM 87301, USA (2002).

27. A. Vadivel and C. John Sundar, $\gamma$-Open Sets in Nnc-Topological Spaces, Advances in Mathematics: Scientific Journal, 9 (4) (2020), 2197-2202.

28. A. Vadivel and C. John Sundar, $N_{n c} \delta$-open sets, Submitted.

29. A. Vadivel and P. Thangaraja, e-open sets $N_{n c}$ Topological Spaces, Submitted.

30. A. Vadivel and P. Thangaraja, e-continuous and Somewhat e-continuity in $N_{n c}$-Topological Spaces, Submitted.

31. A. Vadivel and P. Thangaraja, Characterizations of completely $N_{n c}$ (weakly $N_{n c} e$ ) irresolute functions via $N_{n c}$ e-open sets, Submitted.

32. V. Venkateswaran Rao and Y. Srinivasa Rao, Neutrosophic Pre-open sets and Pre-closed sets in Neutrosophic Topology, International Journal of chemTech Research, 10 (10) 449-458.

33. F. Wadei, Al-Omeri and Saeid Jafari, Neutrosophic pre-continuity multifunctions and almost pre-continuity multifunctions, Neutrosophic Sets and Systems, 27 (2019) 53-69. 